\title{
Transaction
}

\section{Studies on the Degraded State of Excavated Archaeological Silk Fibers using Infrared Micro-Spectroscopy and Curve Fitting Analysis}

\author{
Masanori Akada ${ }^{* 1}$, Masanori Sato ${ }^{* 2}$, and Masayoshi Okuyama ${ }^{* 3}$ \\ ${ }^{* 1}$ Division of Advanced Fibro-Science, Graduate School of Science and Technology, Kyoto Institute of \\ Technology, Matsugasaki, Sakyo-ku, 606-8585, Japan \\ ${ }^{* 2}$ Nara National Research Institute for Cultural Properties, 2-9-1, Nijyo-cho, Nara 630-8577, Japan \\ ${ }^{* 3}$ Archaeological Institute of Kashihara, Nara Prefecture, Unebi-cho, Kashihara, Nara 634-0065, Japan
}

\begin{abstract}
The almost archaeological silk fibers are heavily degraded due to the long-term preservation at underground. The authors have been continuing the scientific research on the degraded state of silk fibers using infrared microspectroscopy. Though we have previously reported results about the characteristics of deformed infrared spectra by curve fitting procedure of the secondary components of fibroin molecules, obtained results were not completed. In the present report, we used the modified procedure to get more reasonable peak top wavenumbers for curve fitting using the second and fourth derivative spectrum of sample infrared spectrum.

The refined silk fiber (Bombyx mori) was used as a modern reference material. The samples of degraded silk fibers were excavated from Fujinoki tumulus $\left(6^{\text {th }} \mathrm{C} . \mathrm{AD}\right)$ and Shimoikeyama tumulus $\left(3^{\text {rd }} \mathrm{C} A D\right)$ in Nara Pref. in Japan.

As a result of curve fitting analysis, it was found that the Amide I peak of the reference silk fiber is consisted of eleven component peaks. The strong component peaks are at $1659 \mathrm{~cm}^{-1}$ (amorphous) and $1650 \mathrm{~cm}^{-1}(\beta$-sheet).

The infrared spectrum of Fujinoki sample showed thirteen component peaks in Amide I peak. Among them, peak at $1672 \mathrm{~cm}^{-1}$ ( $\beta$-sheet) showed strong intensity compared with that of corresponding reference spectrum. On the other hand, the component peak intensity at $1667-1655 \mathrm{~cm}^{-1}$ (amorphous) is evidently decreased compared with that of corresponding reference spectrum. Namely it was shown that the crystallinity of fibroin molecules increases in the degraded state.
\end{abstract}

(Received 27 May, 2009; Accepted 22 June, 2009)

\section{Introduction}

Several kinds of textile fabrics made of silk fibers were found from many archaeological sites in Japan. Almost all of them were however heavily degraded due to the long-term preservation at underground. Therefore, the identification of textile materials is often difficult. Besides, the sampling amount for the scientific analysis is usually limited in minimum.

The FT-IR microscope is one of the versatile instruments for the identification of textile materials for cultural properties. In the course of the continuous research on the identification of the textile materials, we are sometimes aware of the deformed infrared spectrum in the case of degraded silk fibers compared with that of the reference silk fibers. The most remarkable deformation was observed in the strong Amide I and Amide II peaks characteristic of protein molecules. Both peaks seem to merge gradually each other in degraded state and finally show the superposed round one peak in heavily degraded state.

Since the secondary structure of silk fibroin is composed of a few structures such as $\beta$-sheet, $\alpha$ helix, and random form, the each peak in the infrared spectrum is composed of several component structures[1], [2]. To separate each component contained in one absorption peak, curve fitting analysis is one of the suitable analytical procedure.

Though the curve fitting analysis used in this report is explained in more detail in $2-4$, the objective for the research is mainly focused on the analysis of Amide I peak of silk fibers. The reason for the deformation of the Amide I peak following the degradation is explained by the various changes in the secondary component peaks.

\section{Sample and analytical procedure}

\subsection{Analytical instruments}

To identify the fiber material, the stereomicroscope and the super high-resolution scanning electron 
microscope (Hitachi) SU-70(SEM) were used. Throughout the study by infrared spectroscopy, the infrared micro-spectroscope (BL 43 IR) of Japan Synchrotron Radiation Research Institute (JASRI) was used. Since the infrared light source of high brilliance is supplied by synchrotron radiation beam (Spring-8), the light beam can sharply focus on the surface of very small amount of sample fibers. The research was continued during 2005- 2009 years under the permitted research subject concerned with the study on the degraded state of archaeological silk fiber [3].

\subsection{Sample silk fibers}

The degraded archaeological silk fibers of Fujinoki tumulus $\left(6^{\text {th }}\right.$ C. AD) were offered by Kashihara Archaeology Institute, Nara Prefecture. The degraded silk fibers showed diverse characteristics : collapsed fibers, absolutely pulverized, etc. At first, the suitable fiber samples were selected under the stereo- microscope. The all of the selected fibers were able to distinguish the direction of fiber axis, and showed clear cross-sectional pattern.

As a preliminary identification of fiber material, the infrared spectrum of sample was measured. Since all of them showed the distinct absorption peaks of secondary amide, the sample was no doubt animal fibers.

The observation using scanning electron microscope showed that the diameter of cross-section of fibers was about 20 micrometer, and their forms were oval or distorted triangle. In summary, the fiber material was identified as silk even in degraded state.

To investigate the degraded state of silk fibers, the modern reference silk fibers (refined) stored in the authors' laboratory were selected as a reference material, since it is known that their infrared spectra are almost very similar each other irrespective of silk cocoon species.

\subsection{Analytical procedure}

Prior to measurement, silk fibers were pressed on the diamond cell to make a thin film sample. Prepared sample on the diamond cell was transferred to the measurement compartment, and measured by transmittance mode. The measurement conditions are as follows : resolution $4 \mathrm{~cm}^{-1}$, scan numbers 1024 or 2048, wavenumber range 4000$700 \mathrm{~cm}^{-1}$.

\subsection{Curve fitting procedure}

The infrared spectrum of silk fibers generally consists of several absorption peaks in which each peak are further composed of the superposed component peaks.

The objective of curve fitting analysis is to decompose the absorption peak into second structure components. It is assumed that the one absorption peak is

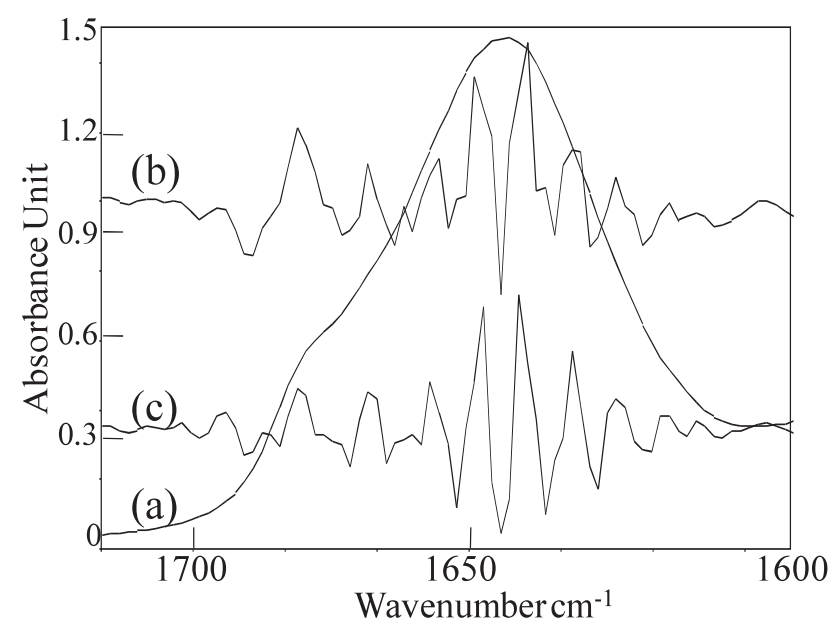

Fig. 1 The spectrum of reference silk at the Amide I region.

(a) IR spectrum of reference silk (b) Second derivative spectrum (c) Fourth derivative spectrum

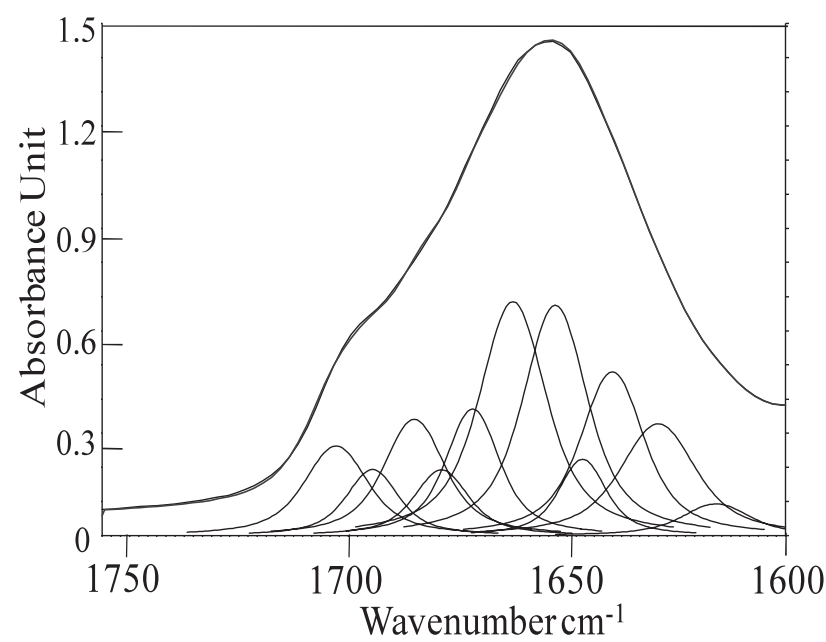

Fig. 2 The curve fitting analysis of reference silk showed eleven component peaks in Amide I peak.

Table 1 The assignment of component peaks in Amide I region for reference silk.

\begin{tabular}{ccc}
\hline Wavenumber $\mathrm{cm}^{-1}$ & Intensity & Secondary structure \\
\hline 1697 & 5.8 & \\
1682 & 5.6 & $\beta$-sheet/disorder \\
1672 & 3.9 & \\
\hline 1668 & 6.1 & $\alpha$-helix/amorphous \\
1657 & 9.0 & \\
\hline 1649 & 9.5 & \\
1637 & 7.3 & \\
1628 & 5.3 & \\
1617 & 4.8 & \\
1610 & 4.5 & \\
\hline 1597 & 4.5 & \\
\hline
\end{tabular}


constructed of the sum of component peaks assigned as a few kinds of secondary structures of fibroin.

In this report, the research interest was mainly focused on the Amide I peak in spectrum of silk fibers. To investigate the change in Amide I peak of degraded silk fibers, the effective procedure is to understand the change in secondary structure components in Amide I peak by curve fitting analysis [4], [5].

At first, it is necessary to set up the fundamental information about each component: peak intensity, peak position (peak top wavenumber) and half-width of component curve. The peak position is decided by the second derivative of original spectrum. If necessary, the fourth derivative [6] of original spectrum should also be refered. To get the second derivative spectrum, the data points was set to $\mathrm{N}=5,7,9,11$ for the convolution calculation. As it was found that the case of $\mathrm{N}=5$ is the best to explain the second structure of silk fibroin, further calculation was performed by using $\mathrm{N}=5$. The next process is to set up the peak intensity and half-width value. The peak intensity is estimated by the relative intensity ratio of component peaks in second derivative spectrum. A small adjustment was made to peak intensity for well adapting to the original Amide I spectrum. The half-width value is also obtained by pattern of second derivative spectrum.

In the course of curve fitting calculation, the computer software selected Voigt function [7] instead of Gauss or Lorentz function [8], [9], as it is well known that the curve fitting analysis of solid sample such as silk fiber does not work well by single use of Gauss or Lorentz function. The use of Voigt function, convoluted form of both Gauss and Lorentz function, was proved to be satisfactory in our study.

At the final stage of set up combining three factors, the numerical values are slightly adjusted to get well fitted result for the reconstruction of original spectrum.

\section{Results and Considerations}

\subsection{Modern reference silk fiber}

As the reference silk, modern refined silk fibers were used. Fig.1 shows the spectrum of reference silk at the Amide I region and its derivative spectrum. The results obtained by the curve fitting analysis of reference silk showed eleven component peaks in Amide I peak. (Fig.2)

The assignment of component peaks in Amide I region is summarized in Table.1. Peaks at higher wavenumber region $\left(1697-1672 \mathrm{~cm}^{-1}\right)$ were assigned as $\beta$ sheet/disorder. $\beta$-sheet means the part where straight

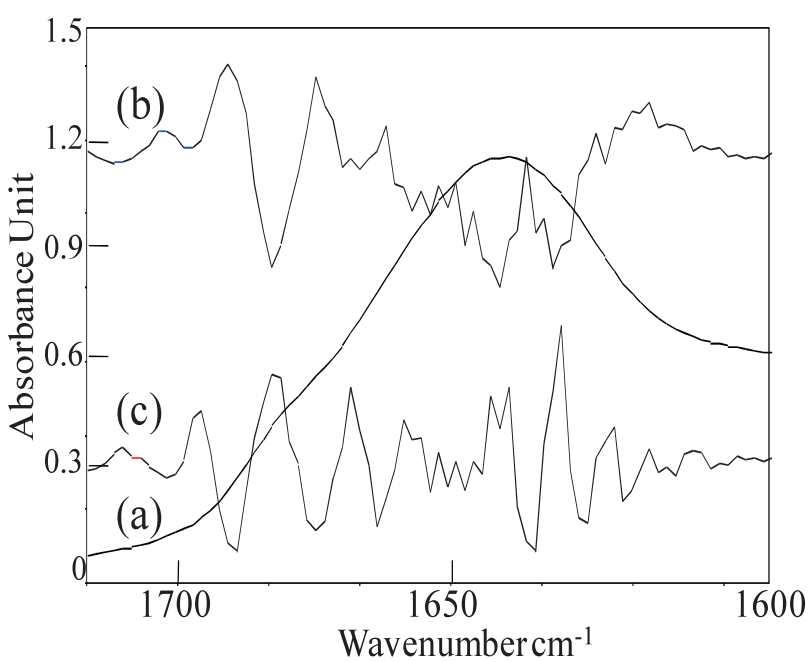

Fig. 3 The spectra of Fujinoki sample fiber at the Amide I region.

(a) IR spectrum of Fujinoki sample (b) Second derivative spectrum (c) Fourth derivative spectrum

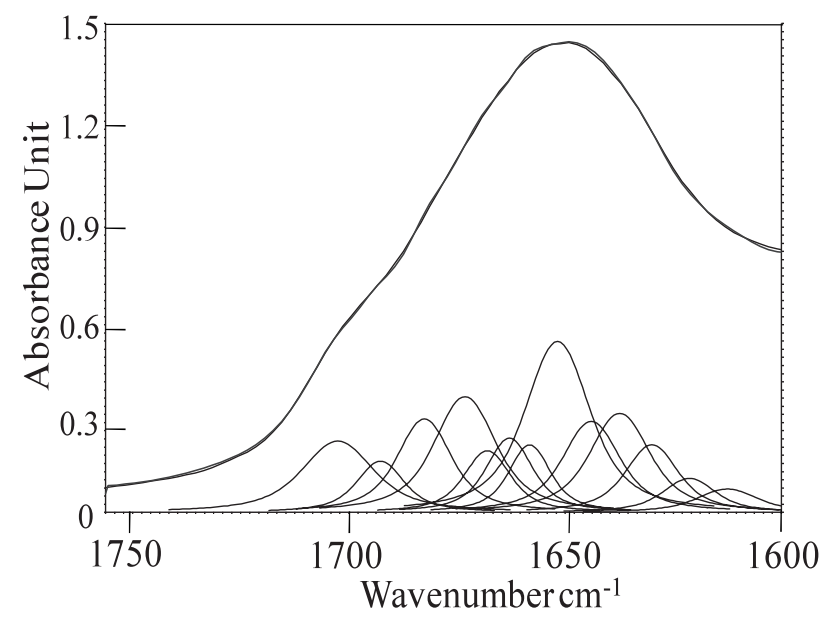

Fig. 4 The curve fitting analysis of Fujinoki sample fiber showed thirteen component peaks in Amide I peak.

Table 2 The assignment of component peaks in Amide I region for Fujinoki sample fiber.

\begin{tabular}{ccc}
\hline Wavenumber $\mathrm{cm}^{-1}$ & Intensity & Secondary structure \\
\hline 1699 & 7.6 & $\beta$-sheet/disorder \\
1682 & 7.5 & \\
\hline 1670 & 6.2 & \\
\hline 1667 & 5.7 & \\
1663 & 4.2 & $\alpha$-helix/amorphous \\
1659 & 4.1 & \\
1655 & 4.4 & \\
\hline 1649 & 7.7 & \\
1642 & 4.8 & \\
1636 & 7.1 & $\beta$-sheet/disorder \\
1626 & 5.4 & \\
1616 & 4.7 & \\
\hline 1605 & 5.1 & \\
\hline
\end{tabular}


peptide chains are lined up in parallel in crystalline area. In this report, the two types of $\beta$-sheet (parallel and antiparallel) are not distinguished each other. Besides, the "disorder" means quasi-crystalline area where microcrystalline and semi-crystalline are mixed [10].

The component peaks at middle wavenumber region (1697-1672 $\left.\mathrm{cm}^{-1}\right)$ in Amide I region are assigned as amorphous/ $\alpha$-helix. "Amorphous" means the noncrystalline part where peptide chains exist randomly. " $\alpha$ helix" means helical crystalline part. In summary, "amorphous" and " $\alpha$-helix" components is difficult to distinguish each other, hence, their peaks are classified as “amorphous/ $\alpha$-helix" [11].

In Fig.2. it is shown that the reference silk has strong component peaks at $1659 \mathrm{~cm}^{-1}$ (amorphous) and $1650 \mathrm{~cm}^{-1}$ ( $\beta$-sheet). This reference silk seems contains relatively much amount of non-crystalline part.

\subsection{Degraded sample of Fujinoki tumulus}

Fig. 3 shows the Amide I region of spectrum of degraded silk and derivative spectrum. ((a) spectrum of degraded sample, (b) corresponding second derivative spectrum, (c) corresponding fourth derivative spectrum.)

As a result of similar analytical procedure with reference sample, thirteen component peaks were recognized in degraded Amide I peak as shown in Fig.4. The increase in the number of component peaks compared with that of reference spectrum is due to the degradation of silk fibers. The assignment of component peaks is summarized in Table.2. The most intensive peak is at $1649 \mathrm{~cm}^{-1}(\beta$-sheet $)$, and hence the ratio in crystalline part is increased compared with that of reference sample.

As for degraded samples, the intensity of three amorphous peaks $\left(1663,1659\right.$ and $1655 \mathrm{~cm}^{-1}$ ) were relatively low compared with that of reference silk. Though the peak $\left(1670 \mathrm{~cm}^{-1}\right)$ is relatively strong, it is rather difficult to decide its assignment, because the peak wavenumber is between $1672 \mathrm{~cm}^{-1}(\beta$-sheet/disorder) and $1668 \mathrm{~cm}^{-1}$ ( $\alpha$-helix/amorphous) of reference silk, besides the peak intensity $\left(1670 \mathrm{~cm}^{-1}\right)$ is comparable with the sum of peak $\left(1672 \mathrm{~cm}^{-1}\right)$ and peak $\left(1668 \mathrm{~cm}^{-1}\right)$. The two peaks (1649 and $1636 \mathrm{~cm}^{-1}$ ) assigned as $\beta$-sheet structure are influenced differently in long-term preservation, though the change in relative peak intensity ratio is not clear.

\subsection{Degraded sample of Shimoikeyama tumulus}

Fig.5 shows (a) the Amide I and II region of spectrum of degraded silk fibers, (b) second derivative spectrum, (c) fourth derivative spectrum, respectively. Since infrared spectrum does not show distinct border between Amide I and Amide II peaks, curve fitting analysis was performed by covering both wavenumber

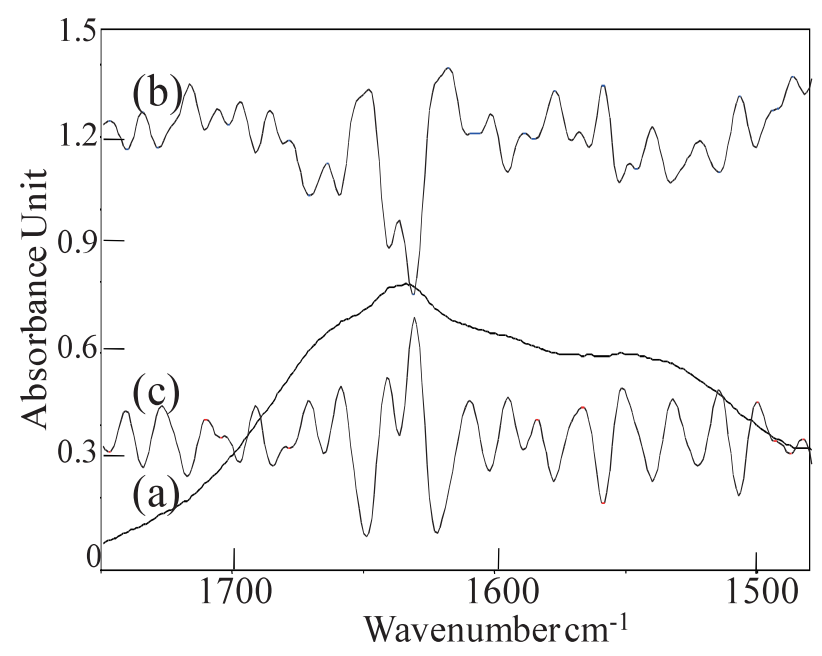

Fig. 5 The spectra of Shimoikeyama sample fiber at the Amide I and II region.

(a) IR spectrum of Shimoikeyama sample fiber

(b) Second derivative spectrum

(c) Fourth derivative spectrum

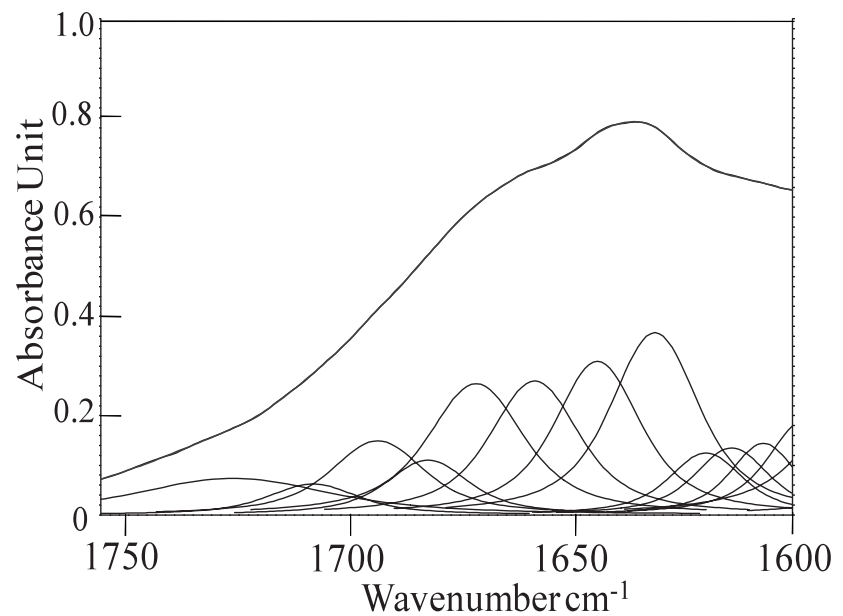

Fig. 6 The curve fitting analysis of Shimoike sample fiber showed eleven component peaks in Amide I peak.

Table 3 The assignment of component peaks in Amide I region for Shimoikeyama sample fiber.

\begin{tabular}{ccc}
\hline Wavenumber $\mathrm{cm}^{-1}$ & Intensity & Secondary structure \\
\hline 1702 & 5.0 & \\
1691 & 6.1 & $\beta$-sheet/disorder \\
1681 & 4.8 & \\
\hline 1672 & 5.3 & \\
\hline 1658 & 6.8 & $\alpha$-helix/amorphous \\
1653 & 3.6 & \\
\hline 1641 & 8.0 & \\
1631 & 8.7 & $\beta$-sheet/disorder \\
1620 & 3.7 & \\
1612 & 5.5 & \\
\hline 1606 & 4.6 & \\
\hline
\end{tabular}


ranges of Amide I Amide II peaks. Fig.6 is the result of curve fitting result at Amide I peak region.

The assignment of component peaks is summarized in Table 3. The most intensive peak $\left(1631 \mathrm{~cm}^{-1}\right)$ was assigned as $\beta$-sheet structure [12], hence it signifies the increase in crystalline part following the progress in degradation. As for the degraded samples, peak intensities at $1702 \mathrm{~cm}^{-1}$ (Anti-parallel $\beta$-sheet) and $1681 \mathrm{~cm}^{-1}$ ( $\beta$-sheet/ disorder) are lower compared with those of reference silk at corresponding peaks. The assignment of peak at $1672 \mathrm{~cm}^{-1}$ is not possible, because its peak seems the broad superposition of two peaks at 1672 and $1668 \mathrm{~cm}^{-1}$. Some of the peaks assigned as amorphous exist at 1658 and $1653 \mathrm{~cm}^{-1}$. The intensity of both peaks are equivalent, and lower than $1631 \mathrm{~cm}^{-1}(\beta$-sheet). Some of the Shimoikeyama sample ( $\beta$-sheet type) showed low intensity due to the degradation, in other word, a part of crystalline part seems collapsed with progress in degradation. At the same time, a part of amorphous component at another secondary structure seems collapsed.

\section{Conclusion}

The change in the secondary structure of degraded silk fiber was clarified by the curve fitting analysis of infrared spectrum depending on the derivative spectrum. In the course of curve fitting procedure, the final setting of necessary information was slightly adjusted by empirical knowledge. As the sum of each component curve was sufficiently coincided with the original spectrum, the difference in two kind of excavated silk fibers kept in different environmental conditions was clarified.

According to results of the curve fitting analysis of Amide I peak, it was ascertained that the secondary structure of degraded silk fiber was evidently different compared with that of modern reference silk fiber. The component spectra of modern reference silk fiber have the strong amorphous peaks compared with that of the crystalline component at 1649 and $1628 \mathrm{~cm}^{-1}(\beta$-sheet/ disorder). Whereas, the relative intensity ratio of the above-mentioned peaks of degraded silk fiber was reversed, namely the amount of crystalline component increased as a result of degradation of silk fibers.

Although, Shimoikeyama's sample apparently seems heavily degraded compared with Fujinoki's sample according to the comparison of both infrared spectra, the comparison of both component spectra showed that the ratio in crystalline part has higher value in Shimoikeyama's sample compared with Fujinoki's sample.

It was concluded that the crystalline part increases following the degradation, and decreased further in heavily degradation. In conclusion, the curve fitting analysis using derivative spectrum was useful to clarify the detail in degraded state of archaeological silk fibers.

The results of this research contribute not only to the identification of degraded fiber material, but also to the conservation treatment of degraded silk fibers.

\section{References}

1. T. Miyazawa, Kagaku to Kogyo, 15, 137 (1962).

2. K. Hirabayashi, H. Ishikawa, M. Kakudo,Y.Go, Sen'i Gakkaishi, 24, 8, 392 (1968).

3. M. Sato, JASRI (Spring-8) (BL43IR), (2005-2008) Applied Subject Number : 2005B0500,2006A1030, 2007A1045, 2007B1174, $2008 A 1249$.

4. M. Sato, Y. Sasaki, "Scintific Analysis of Ancient and Historic Textiles", University of Southampton, 44 (2005).

5. M. Sato, "Historic Silks" (Kinu bunkazaino sekai), Nara national research Institute for cultural properties, (Ed. M. Sato), 3-4, Infrared microspectroscopy, 145 (2005).

6. M. Iwamoto, J. Uozumi, Nippon Shokuhin Kogyo Gakkaishi, 32, 9, 685 (1985).

7. T. Ichikawa, H. Terada, Yakugakuzasshi, 103(8), 878 (1983).

8. T. Ohno, C. Umeno, F. Hatayama, Y. Toda, H. Miyata,J. Chem. Software, 6, 2, 55 (2000)

9. X. Chen, Z. Shao, N. S. Marinkovicb, L. M. Miller, P. Zhou, M. R. Chance, Biophysical Chemistry, 89, 25 (2001).

10. K. Ashizawa, K. Uchikawa, T. Hattori, Y. Ishibashi, Y. Miyake, T. Sato, Yakugaku zasshi, 110(3), 202 (1990).

11. I. C. Um, H. Y. Kweon, K. G. Lee, Y. H. Park, J. Int. Biological Macromolecules, 33, 203 (2003)

12. K. Ogho, C. Zhao, M. Kobayashi, T. Asakura, Polymer, 44, 841 (2003). 